\title{
Quelques performances reproductives et taux de survie de deux souches du poisson-chat africain Clarias gariepinus (Burchell, 1822) et de leurs croisés à Koupa-Matapit
}

\author{
Claudine Tekounegning TIOGUE ${ }^{1^{*}}$, David NGUENGA ${ }^{2}$, Minette T. Eyango TOMEDI ${ }^{3}$ \\ et Joseph TCHOUMBOUE ${ }^{1}$ \\ ${ }^{1}$ Université de Dschang, Faculté d'Agronomie et des Sciences Agricoles (FASA), Chef de Département des \\ Productions Animales, BP 222 Dschang, Cameroun. \\ ${ }^{2}$ Institut de Recherche Agricole pour le Développement, Unité de Recherche Piscicole,. BP 255 Fouman, \\ Cameroun. \\ ${ }^{3}$ Université de Dschang, Faculté d'Agronomie et des Sciences Agricoles (FASA), Département de Foresterie, \\ BP 222 Dschang, Cameroun. \\ Auteur Correspondant,Tel : +237 75079565,_E-mail: tekou_claudine@yahoo.fr
}

\section{RESUME}

Des essais de croisements réciproques de deux souches [la souche sauvage (SS) et la souche domestique (SD)] du poisson-chat africain Clarias gariepinus, ont été effectués entre août et décembre 2005 à la station piscicole de Koupa-Matapit, à l'Ouest-Cameroun. Les taux de fécondation et d'éclosion ont été significativement plus élevés $(\mathrm{P}<0,05)$ chez le parent sauvage SSXSS $(75,97$ et $74,32 \%)$ et le croisé SSXSD (76,45 et $75,14 \%)$ comparés au parent domestique SDXSD (30,81 et 27,10\%) et au croisé SDXSS (45,40 et $41,36 \%)$. Le taux d'embryons déformés a été significativement plus élevé $(\mathrm{P}<0,05)$ chez la souche domestique $\operatorname{SDXSD}(6,44 \%)$ comparé aux autres groupes génétiques SSXSS (1,67\%), SSXSD (2,33\%) et SDXSS (3,57\%). Le taux de survie des larves a varié, chez SSXSS, SDXSD, SSXSD et SDXSS respectivement comme suit : au $\mathrm{J} 3(96,66 ; 86,00 ; 84,00$ et $89,33 \%)$, J20 $(46,26 ; 48,66 ; 35,50$ et $64,80 \%)$ et $\mathrm{J} 90(44,16 ; 31,25 ; 32,50$ et $42,50 \%)$. Les différences n'étaient pas observées à tous les âges ( $>>0,05)$, sauf chez le croisé SDXSS au J20 $(\mathrm{P}<0,05)$. A certains stades de développement, les hétérosis de survie ont été positives. Les croisements intraspécifiques, et l'élevage en hapas pourront ainsi contribuer à améliorer la production des juvéniles de $C$. gariepinus.

(C) 2008 International Formulae Group. All rights reserved.

Mots clés : croisement intraspécifique, fécondation, éclosion, survie, hétérosis, Cameroun.

\section{INTRODUCTION}

Produire massivement des poissons de bonne qualité capables de survivre à des variations des conditions de l'environnement reste le souci majeur de tous les pisciculteurs. En aquaculture, les sélections (sur pedigree, sur descendance, massale et familiale), les manipulations polyploïdies (exemple : triploïdie et tétraploïdie), les croisements interspécifiques et intraspécifiques sont fréquemment utilisés dans le cadre de l'amélioration des performances d'élevage (Dunham et al., 1977, Wohlfarth, 1993). Les croisements interspécifiques chez les poissons peuvent produire des hybrides aux caractéristiques appréciables telles que la stérilité, les populations monosexes, l'hétérosis pour les taux de croissance et de survie, pour la résistance aux maladies... (De Graaf et Janssen, 1996). C'est ainsi que des hybridations interspécifiques et intergénériques ont réussi en Asie chez les 
siluridés, notamment entre Clarias macrocephalus et Clarias batrachus (Boonbrahm et al., 1977), Clarias batrachus ou Clarias macrocephalus et Pangasius hypophthalamus (Tarnchalanukit, 1986), et Clarias batrachus et Heteropneustes fossilis (Mukhopadathy et Dehadrai, 1987). En Thaïlande, il a été produit avec succès par le croisement artificiel d'une femelle de Clarias macrocephalus avec un mâle de Clarias gariepinus des hybrides à croissance rapide et résistant à des maladies plus que d'autres hybrides (Tonguthai et al., 1993). Au Bangladesh, des hybrides entre Pangasius hypophthalamus et Clarias gariepinus ont été mis sur le marché (De Graaf et Janssen, 1996). En Afrique, des hybrides entre Clarias gariepinus et Heterobranchus longifilis ont pu voir le jour (Legendre et al., 1992). Ces auteurs ont rapporté que ces hybrides réciproques étaient viables, leurs taux de survie étaient similaires à ceux des parents maternels, les taux de croissance de Heterobranchus longifilis et des hybrides étaient plus élevés comparés à ceux de Clarias gariepinus.

Les valeurs positives de l'hétérosis ont été aussi produites à travers des croisements intraspécifiques chez de nombreuses espèces, notamment les salmonidés (HörstgenSchwark et al., 1986)), les cichlidés (Tave et al., 1990), les cyprinidés (Wohlfarth, 1993) et les siluridés (Dunham et al., 1990). Au Cameroun, les valeurs positives d'hétérosis ont été obtenues dans les croisements réciproques entre une souche sauvage et une souche domestique de Heterobranchus longifilis Valenciennes (1840) en conditions contrôlées; les taux de survie des croisés à tous les stades de développement étaient intermédiaires à ceux des parents (Nguenga et al., 2000). La vigueur hybride peut également être produite par des croisements réciproques entre les souches de Clarias gariepinus. En effet Clarias gariepinus, grâce à sa rusticité, son régime alimentaire omnivore, sa croissance rapide, sa chair très appréciée et son potentiel commercial élevé intéresse généralement les pisciculteurs et les consommateurs camerounais. Cependant, la disponibilité et la qualité des juvéniles pour l'approvisionnement des étangs d'élevage reste un handicap sérieux.
A notre connaissance, dans la littérature il n'existe aucune donnée sur l'amélioration des performances d'élevage du poisson-chat africain Clarias gariepinus à travers les croisements intraspécifiques en conditions africaines. C'est à cet effet qu'un essai de croisements réciproques a été conduit dans le but d'évaluer les taux de fécondation, d'éclosion, d'embryons déformés à l'éclosion et de survie de deux souches sauvage et domestique de cette espèce et de leurs croisés réciproques à la station piscicole de KoupaMatapit.

\section{MATERIEL ET METHODES \\ Origine et maintenance des géniteurs}

Deux souches du poisson-chat africain Clarias gariepinus (Burchell, 1822) ont été utilisées dans les essais d'évaluation des souches et des croisements intraspécifiques à la Station de Recherche Piscicole de KoupaMatapit de l'IRAD (l'Institut de Recherche Agricole pour le Développement) de Foumban (LN : $5^{\circ} 21^{\prime}$ à $5^{\circ} 58^{\prime}$ et LE : $10^{\circ} 17^{\prime}$ à $11^{\circ} 02^{\prime}$, température moyenne de l'eau $22{ }^{\circ} \mathrm{C}$ ) dans l'Ouest Cameroun. La souche sauvage (SS) était constituée des poissons pêchés de la rivière Nkam au lieu dit Konsoung dans Melong; LN : $5^{\circ} 20^{\prime}$ et LE : $9^{\circ} 53$ ', altitude moyenne $<1200 \mathrm{~m}$, températures moyennes de l'air $22{ }^{\circ} \mathrm{C}$ et de l'eau $21{ }^{\circ} \mathrm{C}$ (Encarta, 2004). Des juvéniles des deux sexes de $10 \mathrm{~g}$, d'âge inconnu ont été introduits dans la station en février 2005. La souche domestique (SD) comprenait des géniteurs, descendants du croisement d'un seul mâle et d'une seule femelle, donc fortement consanguin, puis de leur progéniture, par des reproductions artificielles à la station. Ils avaient été pêchés de la rivière Mapé à Bankim par Foumban : LN $5^{\circ} 21^{\prime}$ à $5^{\circ} 58^{\prime}$ et LE $10^{\circ} 17^{\prime}$ à $11^{\circ} 02^{\prime}$ Température moyenne de l'eau $20,5{ }^{\circ} \mathrm{C}$, altitude moyenne $1145 \mathrm{~m}$ (Encarta, 2004); et introduits dans la station depuis 1987 dans le cadre du projet CRDI (Centre de Recherche/Développement International) Canada de pisciculture intégrée (Piscicultureaviculture) pour le développement de la pisciculture au Cameroun. Les poissons de chaque souche étaient élevés dans un étang de terre de $400 \mathrm{~m}^{2}$ alimenté d'eau en provenance d'un lac artificiel situé en amont jusqu'à la maturité sexuelle. Ils étaient nourris une fois par jour (8 h 00) d'aliment local composé 
(35\% de protéines brutes) à $1 \%$ de leur poids corporel jusqu'au début de l'essai.

\section{Sélection des géniteurs}

Sept femelles de chaque souche, présentant chacune un ventre gonflé et mou, une papille génitale protubérante, rougeâtre ou rosâtre, et dont les ovocytes de diamètre supérieur à $1 \mathrm{~mm}$ et de couleur verdâtre s'obtenaient facilement par pression manuelle légère de l'abdomen, de poids corporel compris entre 300 à $400 \mathrm{~g}$ pour la souche sauvage et 350 à $500 \mathrm{~g}$ pour la souche domestique, ont été sélectionnées. Elles ont été gardées individuellement à jeun pendant 24 heures dans des bacs en plastiques de 601 , numérotés en fonction de la souche, et connectés à un système de renouvellement d'eau à un débit de $200 \mathrm{ml} / \mathrm{mn}$.

Sept mâles de chaque souche, de poids corporel compris entre 275 et $350 \mathrm{~g}$ (souche sauvage), 400 et $450 \mathrm{~g}$ (souche domestique) ont été sélectionnés en fonction de leur papille génitale bien développée et de leur poids corporel. Ils ont été gardés dans un même bac en béton dans l'écloserie. Un thermomètre à mercure mini-maxi était placé dans un des bacs pour le contrôle de la température de l'eau.

\section{Induction hormonale}

Le lendemain de la pêche dans les étangs, après avoir pesé chaque femelle à l'aide d'un peson à ressort de précision $0,5 \mathrm{~g}$, la maturation ovocytaire finale et l'ovulation ont été induites par une injection intramusculaire de l'hormone HCG à la dose de 4000 UI/kg de poids corporel. Chaque femelle ainsi traitée était remise dans son bac. La température de l'eau et l'heure de l'injection ont été notées. Les mâles n'ont reçu aucune injection.

\section{Récolte des gamètes}

La laitance a été collectée suivant la technique de la gonadectomie partielle décrite par Nguenga (2000) chez les mâles. Ensuite, les ovules obtenus par pression manuelle de l'abdomen des femelles selon l'ordre d'injection hormonale ont été recueillis dans des bols en plastique bien secs ; puis pesés sur une balance (OHAUS .GA : 110 ; précision de $0,1 \mathrm{~g})$. Les ovules des femelles de chaque souche ont été mélangés; de même que les laitances des mâles de la même souche.

\section{Conduite de l'essai}

Dans les croisements réciproques, la femelle a été nommée la première. Pour produire les types génétiques purs (SSXSS et SDXSD) et les croisés réciproques (SSXSD et SDXSS), la laitance était prélevée à l'aide d'une seringue et mélangée aux ovules (prélevés à l'aide d'une spatule en inox). La fécondation était faite par addition de quelques ml d'eau de l'écloserie au mélange, suivie d'une agitation modérée pendant $1 \mathrm{mn}$. Les oeufs fertilisés étaient ensuite rincés à l'eau pour enlever l'excès de la laitance, le moment d'addition d'eau aux œufs était noté comme l'heure de fécondation.

Essai 1: Taux de fécondation, d'éclosion, d'anomalie et de survie des larves.

Pour chaque croisement, 3 groupes de $0,2 \mathrm{~g}$ d'ovules (100 à 140 ovules) ont été fécondés chacun avec $1 \mathrm{ml}$ de laitance tel que décrit précédemment et incubés dans 3 bols plastiques de $100 \mathrm{ml}$ contenant $50 \mathrm{ml} \mathrm{d}$ 'eau d'écloserie ; 1 bol contenant 0,2 g d'œufs non inséminés et mélangé à l'eau était préparé pour chaque croisement et a servi de témoin. Le temps mis par les œufs du bol témoin pour devenir blancs était noté et après quoi tous les autres œufs ont été considérés comme fécondés et leur nombre a permis d'évaluer le taux de fécondation. La température de l'eau a permis de définir la durée d'incubation après laquelle le taux d'éclosion de chaque groupe génétique était évalué. Une quantité de $0,5 \mathrm{~g}$ d'œufs de chaque groupe génétique a été inséminée de la même manière que précédemment, et a été respectivement incubés sur des cadres grillagés en bacs de 60 1 et dans des bassins en polyester ; les larves obtenues ont été utilisées dans la suite des essais. Les œufs fécondés et les larves écloses (J0) de chaque groupe expérimental étaient dénombrés par observation directe à la lumière du jour.

Pour évaluer le taux de survie à la fin de la résorption vitelline, 50 larves normales issues de chaque lot expérimental ont été ensuite remises dans leur bol respectif. Les bols ont été placés dans un bac plastique de 60 1 alimenté par un système de tuyauterie en PVC installé en parallèle et muni de robinet. Grâce à un système de contrôle, l'eau était 
maintenue à un niveau constant de $24 \pm 0,50$ cm par un tuyau de trop - plein en PVC; le débit d'eau était de $200 \mathrm{ml} / \mathrm{mn}$. Ces bols flottaient dans le bac et l'eau y était renouvelée 3 fois par jour. Le moment à partir duquel les larves ont manifesté un comportement de recherche et de prise d'aliment, c'est-à-dire peu avant la fin de la résorption vitelline (J3), a été considéré comme le temps de première alimentation exogène et à ce moment, les larves restant dans chaque bol ont été comptées.

Les taux de fécondation (nombre d'œufs fécondés /nombre total d'œufs incubés) X 100), d'éclosion (nombre total de larves écloses / nombre total d'œufs incubés) $X$ 100), le taux de larves déformées (nombre de larves déformées / nombre total de larves écloses) X 100 étaient calculés.

Essai 2 : Taux de survie des alevins.

Pour chacun des croisements parentaux (SSXSS, SDXSD) et croisés (SSXSD et SDXSS), 3 groupes de 250 larves âgées de 3 jours post-éclosion ont été repartis au hasard dans 3 hapas $(0,5 \times 0,5 \times 0,7 \mathrm{~m})$ de mailles 0,5 $\mathrm{mm}$, fixés à l'aide des piquets dans un étang de terre de $276 \mathrm{~m}^{2}$ de la station. L'étang était fertilisé avec $10 \mathrm{~kg} / \mathrm{m}^{2}$ de fientes de poules et $0,8 \mathrm{~kg} / \mathrm{m}^{2}$ d'engrais $20: 10: 10$, trois jours avant le début de l'expérience. Les larves ont été nourries à $40 \%$ de leur poids corporel 2 fois par jour ( $8 \mathrm{~h}$ et $18 \mathrm{~h}$ ) avec un aliment composé des farines d'écrevisses (40\%), de poisson $(45 \%)$, de maïs $(9 \%)$ et du manioc (1\%), de l'huile de soja (3\%) et du prémix vitaminique (1\%). L'analyse chimique de cet aliment donne la composition suivante : protéines brutes $(48,55 \%)$, cellulose brute $(27,81 \%)$, lipides $(12,64 \%)$, sels minéraux $(6 \%)$ et vitamines $(5 \%)$. Tous les 4 jours, les poissons de chaque hapa étaient comptés et transférés dans un seau de 10 litres, et le hapa était lavé puis replacé à son emplacement dans l'étang. Les poissons étaient ensuite retournés dans leur hapa respectif. Au dernier jour de l'élevage (J20 après éclosion) chaque hapa était détaché et retiré de l'étang, les survivants étaient comptés.

Essai 3 : Taux de survie des juvéniles.

Pour chacun des croisements SSXSS, SDXSD, SSXSD et SDXSS obtenus de l'expérience 2, 3 groupes de 40 alevins âgés de 20 jours post-éclosion ont été stockés séparément et au hasard dans 12 hapas (1 x
$0,8 \times 0,7 \mathrm{~m}$ ) de mailles $2 \mathrm{~mm}$ placés à l'aide des piquets dans l'étang de terre de l'expérience 2. L'étang a été fertilisé au début de l'essai aux mêmes doses que dans l'expérience 2, la fertilisation s'est répétée une fois par semaine avec des doses réduites de moitié, jusqu'à la fin de l'essai (J90). Les poissons étaient nourris 2 fois par jour $(8 \mathrm{~h}$ et $18 \mathrm{~h}$ ) avec l'aliment composé précédemment décrit, à la même dose pendant les deux premières semaines. La dose journalière a été ensuite doublée jusqu'à la fin de l'expérience. Tous les 14 jours, chaque hapa était traité comme dans l'essai 2. Au bout de 70 jours d'élevage (J90), les hapas ont été vidés et les survivants ont été comptés.

A la fin de chaque essai, le taux de survie (nombre de poissons obtenus en fin d'expérience / nombre de poissons à la mise en charge) X 100 de chaque type génétique a été calculé. Le nombre final moyen des poissons des types génétiques parentaux (P1 et P2) et des croisés (H1et H2) a permis de calculer l'hétérosis : $100 \mathrm{X} \quad[(\mathrm{H} 1+\mathrm{H} 2) / 2$ $(\mathrm{P} 1+\mathrm{P} 2) / 2] /(\mathrm{P} 1+\mathrm{P} 2) / 2$.

Les taux de fécondation et d'éclosion des oeufs; le taux des larves déformées à l'éclosion et les taux de survie ont été comparés par une analyse de la variance à un facteur et lorsque les différences étaient significatives, les moyennes étaient testées deux à deux par le test multiple de Duncan au seuil de $5 \%$.

\section{RESULTATS}

Taux de fécondation, d'éclosion des oeufs et taux d'anomalie des embryons à l'éclosion Taux de fécondation et d'éclosion des oufs : Ces paramètres ont été significativement plus élevés $(\mathrm{P}<0,05)$ chez la souche parentale SSXSS et chez le croisé SSXSD comparés à la souche parentale SDXSD et au croisé SDXSS (P>0,05) (Figure 1).

Taux de déformation des embryons à l'éclosion : Le taux d'anomalie des embryons à l'éclosion a varié entre les groupes génétiques, mais est resté relativement faible (inférieur à 10\%) dans tous les cas (Figure 2). Toutefois, il est significativement plus élevé $(\mathrm{P}<0,05)$ chez le parent domestique SDXSD que chez tous les autres groupes génétiques. 


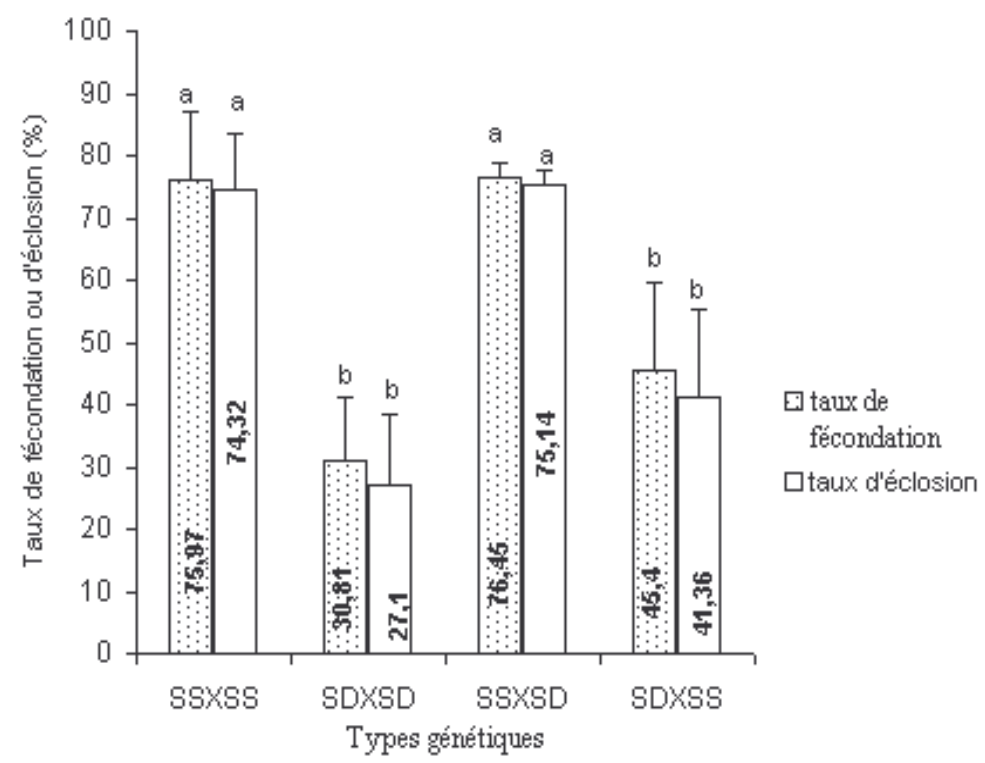

$\mathrm{T}=$ écart type de la moyenne $(\mathrm{n}=3)$

a, b: Les barres verticales portant des lettres semblables ne sont pas significativement différentes $(\mathrm{P}>0,05)$.

Figure 1: Taux de fécondation et d'éclosion de deux souches de C. gariepinus et de leurs croisés réciproques dans les conditions de la station piscicole de Koupa-Matapit, Foumban, Ouest-Cameroun.

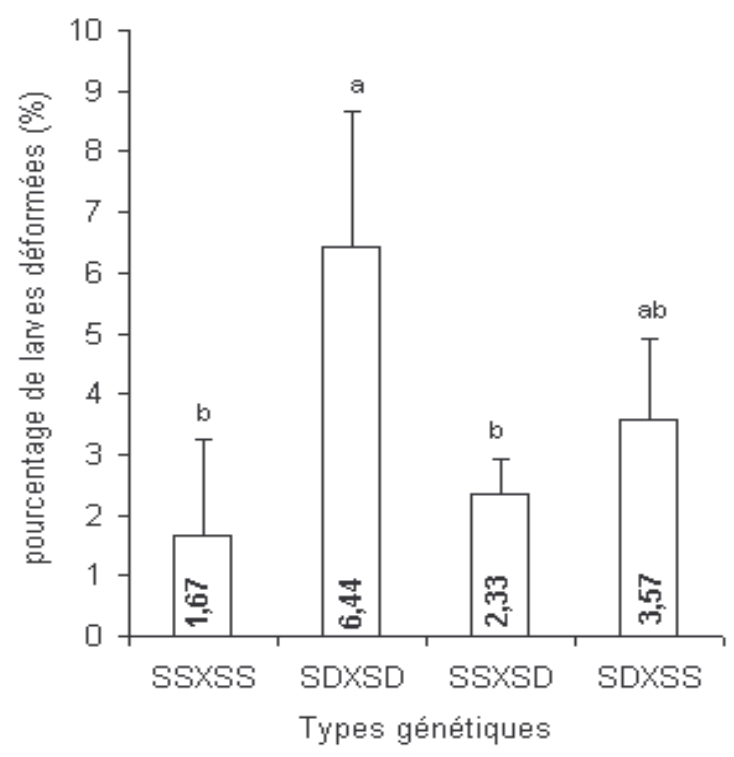

$\top=$ écart type de la moyenne $(\mathrm{n}=3)$

a, b: Les barres verticales portant des lettres semblables ne sont pas significativement différentes $(\mathrm{P}>0,05)$.

Figure 2: Pourcentage d'embryons déformés à l'éclosion de deux souches de $C$. gariepinus et de leurs croisés réciproques dans les conditions de la station piscicole de Koupa-Matapit, Foumban, Ouest-Cameroun. 
Taux de survie des larves à différents stades de développement

A la résorption vitelline: les groupes génétiques étudiés ont des taux de survie comparables ( $\mathrm{P}>0,05$ : Tableau 1), la survie est légèrement plus élevée chez le parent sauvage SSXSS, suivi respectivement du croisé SDXSS, du parent domestique SDXSD et du croisé SSXSD.

Au stade alevin : Il ressort du tableau 1 que le croisé SDXSS a un taux de survie significativement plus élevé $(\mathrm{P}<0,05)$ que celui des autres types génétiques qui sont par ailleurs comparables entre eux $(\mathrm{P}>0,05)$ pour ce paramètre.

Au stade juvénile : A 90 jours d'âge postéclosion, le parent sauvage SSXSS a un taux de survie plus élevé que dans les autres croisements, mais de manière non significative $(\mathrm{P}>0,05)$ (Tableau 1$)$.

\section{Hétérosis de la survie}

Il ressort du tableau 2 que les hétérosis positives de la survie dans les croisements réciproques des deux souches ont été enregistrées aux stades œuf, embryon et alevin.

\section{DISCUSSION}

Taux de fécondation et d'éclosion des oufs et taux de déformation des embryons à l'éclosion

Les taux de fécondation et d'éclosion ont été nettement inférieurs à ceux généralement obtenus chez les Clariidae en conditions contrôlées : 87,1 à $95,2 \%$ chez $H$. longifilis (Nguenga et al., 2000) pour les taux de fécondation, et 90,37 à 92,96\% chez $H$. longifilis (Agnèse et al., 1995) pour les taux d'éclosion. Ces faibles valeurs pourraient être dues à une mauvaise qualité initiale des gamètes (Nguenga et al., 2000). Ces performances d'élevage ont été faibles chez le parent domestiqué comparé au parent sauvage. Chez Heterobranchus longifilis, alors que Nguenga et al. (2000), ont rapporté des résultats similaires, Agnèse et al. (1995) n'ont montré aucune différence significative entre les deux souches. Cela peut s'expliquer par le fait que les souches utilisées dans cette étude soient génétiquement plus éloignées que celles utilisées par ces derniers auteurs. Les gènes qui gouvernent la production de ces performances, transmis au croisé SSXSD ont été si dominants qu'il a enregistré les meilleures valeurs. La dégénérescence génétique aurait aussi conduit à une augmentation d'embryons déformés à l'éclosion et à une réduction d'embryons normaux chez le parent domestique. Ces résultats sont proches de ceux rapportés par Agnèse et al. (1995) chez H. longifilis.

\section{Taux de survie à différents stades de développement}

A la résorption de la réserve vitelline: Le Taux de survie des larves à la résorption vitelline a été élevé (> 80\%) conformément aux résultats habituellement obtenus chez les Clariidae à ce stade de développement. Toutefois ce taux a baissé chez le parent domestique ; ces résultats sont comparables à ceux rapportés par Agnèse et al. (1995) chez $H$. longifilis, différents de ceux rapportés par Nguenga et al. (2000) chez cette même espèce. Cette différence serait due aux facteurs intrinsèques de la souche utilisée. De même que chez Nguenga et al. (2000) les croisés ont enregistrés des taux de survie intermédiaires. Baras et d'Almeida (2001) expliquent que le cannibalisme contribue à la diminution du taux de survie et qu'il est intermédiaire chez les croisés comparés aux parentaux.

Au stade alevin : Les taux de survie obtenus ont été faibles comparés aux résultats de 80 à 96\% rapportés en conditions contrôlées chez les Clariidae (Haylor, 1992 ; Nguenga et al., 2000). La majorité des mortalités surviennent surtout en début de la phase d'alevinage, le facteur critique étant la disponibilité en plancton. En conditions contrôlées, l'aliment vivant Artemia a été utilisé dès les premiers jours d'alevinage, ce qui a produit un taux de survie élevé. Or dans les conditions de cette étude l'étang d'alevinage n'était pas protégé contre les prédateurs et surtout contre les têtards de grenouilles, véritables concurrents pour les aliments (De Graaf et Janssen, 1996 ; Yong-Sulem et al., 2006). De plus, l'aliment fabriqué à base de produits locaux semble n'avoir pas été facilement accessible aux larves sans doute en raison de la taille variable des particules. Ces résultats peuvent aussi s'expliquer par l'effet du colmatage des hapas qui aurait entraîné la mort de certains poissons avant le jour du nettoyage. Toutefois les taux de survie ont été comparables à ceux de 30 à $50 \%$ rapportés par Campbell et al. (1995) 
Tableau 1: Taux de survie à différents stades de développement de deux souches de C. gariepinus et de leurs croisés réciproques dans les conditions de la station piscicole de Koupa-Matapit, Foumban, Ouest-Cameroun.

\begin{tabular}{lcccc}
\hline Stade de & \multicolumn{4}{c}{ Taux de survie (\%) } \\
\cline { 2 - 5 } développement & SSXSS & SDXSD & SSXSD & SDXSS \\
\hline Larves (J0 à J3) & $96,66 \pm 3,05$ a & $86,00 \pm 11,13 \mathrm{a}$ & $84,00 \pm 24,33$ a & $89,33 \pm 4,16$ a \\
Alevins (J3 à J20) & $46,26 \pm 9,31 \mathrm{~b}$ & $48,66 \pm 5,99 \mathrm{~b}$ & $35,50 \pm 2,49 \mathrm{~b}$ & $64,80 \pm 2,43 \mathrm{a}$ \\
Juvénile (J20 à J90) & $44,16 \pm 15,27 \mathrm{a}$ & $31,25 \pm 12,37 \mathrm{a}$ & $32,50 \pm 10,89 \mathrm{a}$ & $42,50 \pm 15,20 \mathrm{a}$ \\
\hline
\end{tabular}

Pour tous les croisements, la femelle a été nommée la première. SS, souche sauvage ; SD, souche domestique.

Les valeurs du tableau sont des moyennes \pm écart type de 3 répétitions. $\mathrm{J}=$ Jour.

a,b : Les valeurs sur une ligne présentant la même lettre ne sont pas significativement différentes $(p>0,05)$.

Tableau 2. Hétérosis de la survie des croisements réciproques entre les souches parentales sauvage (SS) du Nkam et domestique (SD) de la Mapé de C. gariepinus par stade de développement.

\begin{tabular}{lc}
\hline Stade de développement & $\begin{array}{c}\text { Hétérosis de la survie } \\
(\%)\end{array}$ \\
\hline De l'ovocyte à l'œuf & $+14,11$ \\
De l'œuf à l'éclosion (J0) & $+14,86$ \\
Larve (J0 à J3) & $-5,10$ \\
Alevin (J3 à J20) & $+2,69$ \\
Juvénile (J20 à J90) & $-0,53$ \\
\hline \multicolumn{1}{c}{$\mathrm{J}=$ Jour. }
\end{tabular}

dans un étang protégé d'une clôture de papier nylon et fertilisé aux fientes de poules et à ceux de $42,68 \%$ rapportés par Rukera et al. (2005) en bassins et en étangs simulés en absence de compétiteurs et de prédateurs. Quoique relativement faibles, ces taux de survie restent corrects, compte tenu du système d'élevage et surtout des particules de l'aliment utilisé. Par ailleurs, le parent domestique a un taux de survie plus élevé que le parent sauvage, mais non significativement. Cette résistance à ces conditions d'élevage, transmise au croisé SDXSS a été si dominante que son taux de survie a été significativement plus élevé que ceux des autres groupes génétiques.

Au stade juvénile : Les taux de survie obtenus ont été faibles comparés à ceux de 57,1 à $85,8 \%$ rapportés chez $H$. longifilis par Nguenga et al. (2000) en conditions contrôlées et à ceux de 66,8 à 82,7\% rapportés par YongSulem et al. (2006) dans des étangs non équipés de compostière et fertilisés aux bouses de vache. Mais ils ont été nettement plus élevés que ceux trouvés au Cameroun (2,7\%) par Hogendorn (1979), au Congo
Brazzaville (5\%) par de Graaf et al. (1995) et au Kenya (5,8\%) par Obuya et al. (1995) en étangs non protégés et fertilisés régulièrement aux fientes de poules. Ils ont par ailleurs été comparables à ceux de 32,2 à $38,7 \%$ rapportés par De Graaf et al. (1995) en étangs protégés d'une clôture de tôles en aluminium; et à ceux de 24 à $39 \%$ rapportés par Rukera et al. (2005) en étangs simulés et en bassins. La présente étude s'est effectuée dans le même étang non protégé qu'au stade alevin, ce qui a favorisé dés la première semaine la présence dans les hapas et aux alentours de l'étang d'élevage des têtards et adultes d'amphibiens, des serpents et des loutres prédateurs des poissons, ceci est d'ailleurs un inconvénient caractéristique de ce système d'élevage. Cependant les hapas semblent avoir beaucoup réduit l'effet des prédateurs sur la survie des juvéniles. Le taux de survie plus élevé mais non significativement chez la souche sauvage comparée à la domestique est similaire au résultat rapporté par Agnèse et al. (1995) chez les souches de $H$. longifilis, différents de ceux de Nguenga et al. (2000) en aquarium chez cette même espèce. Cette différence 
pourrait être due au fait qu'en aquarium les conditions artificielles sont surtout favorables aux poissons domestiqués.

De même que Nguenga et al. (2000) chez $H$. longifilis, les valeurs positives d'hétérosis enregistrées à certains stades de développement traduirait la supériorité des croisés sur les parents, tandis que la valeur négative exprimerait une interaction négative entre les gènes parentaux qui se serait produite sur les loci des croisés.

\section{Conclusion}

$\mathrm{Au}$ terme de l'étude sur quelques performances reproductives et le taux de survie de deux souches de poisson chat africain Clarias gariepinus (Burchell, 1822) et de leurs croisés à Koupa-Matapit, il convient de retenir ce qui suit :

La souche sauvage SSXSS et le croisé SSXSD ont enregistré les meilleurs taux de fécondation et d'éclosion comparés à ceux des autres types génétiques; la souche domestique a enregistré à l'éclosion le taux d'embryons déformés le plus élevé. Le type génétique n'a influencé le taux de survie qu'au stade alevin. Ainsi, il a été plus élevé chez le croisé SDXSS comparé aux autres types génétiques testés. Des valeurs positives de l'hétérosis ont été enregistrées à certains stades de développement. $\mathrm{Au}$ vu des performances d'élevage enregistrées, ces croisements et ce système d'élevage pourront être efficaces pour l'amélioration de la production des juvéniles de C. gariepinus.

\section{REMERCIEMENTS}

Nous remercions tout le staff de la station piscicole de Koupa-Matapit (IRAD de Foumban, Cameroun) pour leur assistance tant matériel que technique.

\section{REFERENCES}

Agnèse JF, Otémé ZJ, Gilles S. 1995. Effects of domestication on genetic variability, fertility, survival and growth rate in a tropical siluriform: Heterobranchus longifilis Valenciennes 1840. Aquaculture, 131: 197-204.

Baras E, d'Almeida AF. 2001. Size heterogeneity prevails over kinship in shaping cannibalism among larvae of sharptooth catfish Clarias gariepinus. Aquatic Living Resources, 14: 251-256.
Boonbrahm M, Tarnchalanukit W, Suraniranat P. 1977. Experiments on hybridization of fresh-water catfish, Clarias macrocephalus (Ghunter) and Clarias batrachus. Research Report of the Kasetsart University, Bangkok, Thailand, $143 \mathrm{p}$.

Campbell D, Obuya S, Spoo M. 1995. A simple method for small scale propagation of Clarias gariepinus in Western Kenya. Field document $\mathrm{n}^{\circ} 2$, FAO/TCP/KEN/4551, 27 p.

De Graaf GJ, Galemoni F, Banzoussi B. 1995. Artificial reproduction and fingerling production of African catfish, Clarias gariepinus (Burchell, 1822), in protected and unprotected ponds. Aquaculture Research, 26: 233-242.

De Graaf G, Janssen H. 1996. Artificial reproduction and pond rearing of the African catfish Clarias gariepinus in Sub-Saharian Africa. A handbook. FAO Fisheries Technical Paper 362; 73 p.

Dunham RA, Smitherman RO, Goodman RK. 1987. Comparison of mass selection, crossbreeding, and hybridization for inproving growth of Channel catfish. Progressive Fish-Culturist, 49: 293-296.

Encarta Microsoft. 2004. Atlas mondial.

Haylor GS. 1992. Controlled hatchery production of Clarias gariepinus (Burchell 1822): an investigation of tank design and water flow rate appropriate for Clarias gariepinus in hatcheries. Aquaculture and Fisheries Management, 23: 649-659.

Hogendoorn H. 1979. Controlled propagation of the African catfish Clarias lazera. Reproductive biology and field experiments. Aquaculture, 17: 323-333.

Hörstgen-Schwark G, Fricke H, Langholz HJ. 1986. The effect of strain crossing on the production performance in rainbow trout. Aquaculture, 57: 141-152.

Legendre M, Teugels GG, Cauty C, Jalabert B. 1992. A comparative study on morphology, growth rate and reproduction of Clarias gariepinus, Heterobranchus longifilis and their reciprocal hybrids (Pisces: Clariidae). J. Fish Biol., 40: 59-79.

Mukhopadathy SM, Dehadrai PV. 1987. Survival of hybrids between airbreathing catfishes Heteropneustes 
fossilis (Bloch) and Clarias batrachus (Linn.). Matsya, 12-13: 162-164.

Nguenga D, Teugels GG, Ollevier F. 2000. Fertilization, hatching, survival and growth rates in reciprocal crosses of two strains of an African catfish Heterobranchus longifilis Valenciennes 1840 under controlled hatchery conditions. Aquaculture Research, 31: 565-573.

Nguenga D. 2000. Partial gonadectomy in the catfish Heterobranchus longifilis (Teleostei, Clariidae): Regeneration time, quality and quantity of postsurgical sperm production. The Israeli Journal of Aquacuture-Bamidgeh, 4(52): 167-172.

Obuya S, Ochieng J, Campbell D. 1995. Integration of chicken raising and rearing of larval Clarias gariepinus in large ponds. Field document No. 3, FAO/KEN/86/027, 14 p.

Rukera TS, Micha JC, Ducarme C. 2005. Essais d'adaptation de production massive de juvéniles de Clarias gariepinus en conditions rurales. Tropicultura, 4 (23): 231-244.

Tarnchalanukit W. 1986. Experimental hybridization between catfishes of the family Clariidae and Pangasiidae in
Thailand. Environmental Biology of Fishes, 16: 317-320.

Tave D, Smitherman RO, Jayaprakas V. 1990. Estimates of additive genetic effects, maternal genetic effects, individual heterosis, maternal heterosis and egg cytoplasmic effects for growth in Tilapia nilotica . Journal of the World Aquaculture Society, 21: 263-270.

Tonguthai K, Chinabut S, Limsuwan C, Somsiri T, Chanratchakool P, Kanchanakhan S, Mac Rae I. 1993. Handbook of hybrid catfish: Husbandry and health. Aquatic Animal Health Research Institute, Departement of fisheries, Kasetsart University Campus, Jatujak, Bangkok 10900, Thailland, 37p.

Wohlfarth G. 1993. Heterosis for growth rate in common carp. Aquaculture, 113: 3146.

Yong-Sulem S., Tomedi E.T., Mounchili S., Tekeng S. \& Brummett R.E. 2006. Survival of Clarias gariepinus fry in earthen ponds: Effects of composts and leaks. Aquaculture, 260: 139-144. 